stand hergestellt werden können, während bei anderen, wie etwa $\mathrm{Hg}$, ein solcher Zustand unter bestimmten Bedingungen erzeugt und erhalten werden kann, ist auf Grund des Herzfeldschen Metallkriteriums nicht nur verständlich, sie ist vielmehr zu erwarten. Daher kann die Hypothese, daß es grundsätzlich möglich sei, alle Metalle in einem nichtleitenden festen Zustand herzustellen, in dieser umfassenden Weise nicht aufrechterhalten werden. Dagegen erscheint es durchaus wahrscheinlich, daß ein amorpher Zustand aller Metalle, gekennzeichnet durch eine völlig regellose
Anordnung der Metallatome, hergestellt werden kann. Der negative Ausfall der Versuche von Richter in bezug auf die Metalle mit ausgesprochen metallischem Charakter ist einerseits auf die relativ hohe Temperatur seines Schichtträgers zurückzuführen, andererseits auf den Umstand, auf den in diesem Zusammenhang schon wiederholt hingewiesen wurde, daß bei Strukturuntersuchungen mit Elektronenstrahlen durch diese selbst Energiezufuhr erfolgt, wodurch sofort Einschwing- und Platzwechselvorgänge und damit Kristallbildungen ermöglicht werden.

\title{
Schnelle Zustandsänderungen in Aluminium-Mischkristallen
}

\author{
Von Hermann Auer* \\ (Z. Naturforschg. 4 a, 533-540 [1949]; eingegangen am 25. April 1949)
}

\begin{abstract}
Die technisch so bedeutungsvollen Aushärtungsvorgänge in übersättigten AluminiumMischkristallen sind auf besondere Atomanordnungen (sog. Nebenzustände) im Gitter zurückzuführen, die von den Fremdatomen vor bzw. neben der eigentlichen Ausscheidung durchlaufen werden. Ihre Untersuchung mit rein physikalischen, atomaren Eigenschaften (z. B. magnetischer Suszeptibilität), die eine besonders einfache Zuordnung $\mathrm{zu}$ örtlichen Atomanreicherungen ermöglichen, hat zur Annahme von zwei Vorzuständen neben der heterogenen Ausscheidung geführt, die durch eine bestimmte Temperaturbehandlung, das sog. Rückbildungsverfahren, getrennt und auf ihre thermische Stabilität hin untersucht werden können. Die z. Tl. sehr schnell ablaufenden Rückbildungsvorgänge erforderten die Entwicklung einer besonderen Anordnung zum kurzzeitigen Anlassen mit möglichst großer Aufheizgeschwindigkeit (,Aufschrecken“) sowie zur Erzielung definierter Überhitzungen (Wärmestöße).

Aus Suszeptibilitäts- und Härtemessungen ergibt sich, daß der Ablauf der Zustandsänderungen bezüglich des mengenmäßigen Anteils und der thermischen Stabilität der einzelnen Zustände weitgehend von der Geschwindigkeit abhängig ist, mit der die Zwischentemperaturen beim Anlassen durchlaufen werden. Wie die Rückbildungsanalyse an aufgeschreckten Proben zeigt, nimmt bei hoher Aufheizgeschwindigkeit der Warmaushärtungsanteil ab, gleichzeitig aber erhöht sich seine thermische Stabilität. Ausgeprägte Hystereseerscheinungen lassen auf Stabilitätsverschiebungen der atomaren Komplexe innerhalb des Warmaushärtungszustandes schließen im Einklang mit den Keimbildungsvorstellungen, wie sie im Sinne der Beckerschen Auffassung für die Entmischungsvorgänge im Kristallgitter maßgebend sind.
\end{abstract}

$V_{A}$ or nunmehr 15 Jahren haben Gerla ch und A u e ${ }^{1}$ darauf hingewiesen, daß die Untersuchung aushärtbarer Leichtmetall-Legierungen mittels rein physikalischer Eigenschaften, ohne Rücksicht auf ihr technisches Interesse, neue und fruchtbare Gesichtspunkte für die Deutung des Aushärtungsmechanismus erwarten lasse. Wie seither eindeutig klargestellt ist, sind die Aushärtungsvorgänge, die beim Anlassen, beson-

* München 2, Marsstr. 1 a.

1 H. A u e r u. W. Ge r l a ch, Metallwirtsch. Metallwiss., Metalltechn. 13, 871 [1934]. ders aber auch bei Kaltaushärtung übersättigter Mischkristalle eine z. Tl. erhebliche Vergütung der mechanischen Eigenschaften ergeben, auf besondere Atomanordnungen bzw. Anreicherungen der Fremdatome im Grundgitter zurückzuführen, die vor der eigentlichen heterogenen Ausscheidung durchlaufen werden. Auf Grund dieser Vermutung wurde daher damals empfohlen, vor allem solche Eigenschaften zur Untersuchung dieser Ansammlungen im Mischkristall heranzuziehen, die - im Gegensatz zu den Mittelwertsgrößen der mechanischen Eigenschaften - 
Schlüsse auf atomare Veränderungen in Atombereichen zulassen.

Mehrere hieran anschließende Arbeiten ${ }^{2-} \overline{7}$ haben sodann erwiesen, daß vor allem die magnetische Suszeptibilität als ausgesprochen atomare Eigenschaft in besonders einfachem und übersichtlichem Zusammenhang mit den Atomumordnungen während der Aushärtung steht. Unter bewußter Beschränkung auf einfache Legierungssysteme (vorwiegend das binäre System $\mathrm{Al}-\mathrm{Cu}$ ) wurden die verschiedenen Zustandsänderungen beim Übergang vom übersättigten Mischkristall in die heterogene Phase magnetisch wie auch mit anderen physikalischen Eigenschaften ${ }^{8-10}$ eingehend untersucht.

Die zu solchen Messungen herangezogenen Eigenschaften sollten natürlich in möglichst einfacher Beziehung zur Menge des untersuchten Zustandes stehen. Für die magnetische Suszeptibilität legt der logarithmisch lineare Verlauf während der Kaltaushärtung sowie ihre völlige Unempfindlichkeit gegenüber Gefügestörungen und inneren Spannungen diesen einfachen $\mathrm{Zu}$ sammenhang nahe, während die anderen bisher untersuchten Eigenschaften kompliziertere Beziehungen, häufig mit Extremwerten, ergeben. Es wäre eine wichtige Aufgabe, für die verschiedenen Eigenschaften die Anreicherungsfunktionen, d. h. ihren Zusammenhang mit den Atomansammlungen im Mischkristall, quantitativ zu ermitteln. Hiermit könnte dann auch die Frage des „Kohärenzbereiches“ einer Klärung nähergebracht werden, d.h. die Frage nach der Mindestgröße eines angereicherten Gebietes, damit es seiner Größe bzw. Atomzahl entsprechend von der betreffenden Eigenschaft registriert wird. Auch die für die verschiedenen Eigenschaften so wechselnden Inkubationszeiten müßten hier ihren Ausdruck finden. Anhaltspunkte für die Deutung solcher Anreicherungsfunktionen, also für den Mechanis-

2 H. A u e r, Z. techn. Physik 16, 486 [1935]; Metallwirtsch. Metallwiss., Metalltechn. 14, 815 [1935].

3 H. A u e r, Z. Metallkunde 28, 164 [1936].

${ }^{4}$ H. A u er u. K. E. Mann, Z. Metallkunde 28, 323 [1936].

${ }_{5}^{5}$ H. A uer, Z. Metallkunde 30, 48 [1938], H. V. (Vortragsheft der Hauptversammlung München 1938).

${ }^{6}$ H. A u e r, Z. Elektrochem. angew. physik. Chem. 45, 608 [1939].

7 H. A uer u. H. Schroeder, Ann. Physik 37, 137 [1940].

${ }_{8}$ W. Hartnagel, Z. Metallkunde 30, 81 [1938].

9 H. A uer u. K. Siemens, Z. Metallkunde 30, 86 [1938]. mus der Einwirkung der Atomumordnung auf bestimmte Eigenschaften, sind u. a. für die Suszeptibilität ${ }^{11}$ und für den elektrischen Widerstand $^{12}$ gegeben worden.

1. Die verschiedenen Aushärtungszustände in Al-Cu-Mischkristallen

Die Aufnahme der Kinetik bei verschiedenen Temperaturen ergibt, daß die bei der Aushärtung ablaufenden Vorgänge, je nach der Anlaßtemperatur, zu mehreren Zuständen verschiedener thermischer Stabilität führen können.

Im unteren Temperaturbereich weist die experimentell bestätigte Linearität der Beziehung $\ln v=f(1 / T) \quad(v=$ Geschwindigkeit der Zustandsänderung) auf die Existenz eines einheitlichen Zustandes mit konstanter Aktivierungsenergie hin, der für die sog. Kaltaushärtung maßgebend ist (Zustand a, einphasige Entmischung).

Beim Anlassen auf höheren Temperaturen ließ die Abweichung von der Arrhenius-Geraden auf eine gleichzeitige Überlagerung mehrerer Zustände schließen. Eine Analysierung wurde jedoch erst möglich, nachdem es gelang, durch die Entwicklung einer neuen Versuchsmethode, des sog. ,Rückbildungsverfahrens" ${ }^{5,6,7}$, die einzelnen Vorzustände zu trennen und ihre Menge und thermische Stabilität aus der Größe und Geschwindigkeit ihrer Zerstörung (Rückbildung) bei noch höheren Temperaturen zu bestimmen.

\section{Das Rückbildungsverfahren}

Der schon seit längerem, zuerst als „Zusammenbruch der Kaltaushärtung“, bekannte aufschlußreiche Vorgang der Rückbildung bei Temperatursteigerung ist erfreulicherweise in den letzten Jahren immer häufiger zur Untersuchung von Zustandsänderungen herangezogen worden ${ }^{13}$, allerdings meist mit der lediglich qualitativen Feststellung der Rückbildbarkeit eines Zustandes bei einer Temperatur und mit konstanter Rückbil-

10 H. Auer u. W. Gerlach, Naturwiss. 27, 299 [1939].

${ }_{11} \mathrm{H}$. A u e r ${ }^{3}$, S. 174.

12 W. Hartn a g e ${ }^{8}$, S. 85 .

13 W. Geller u. H. Kuntze, Z. Metallkunde 40, 16 [1949]; F. C. A l th of, Metallforschg. 2, 365 [1947]; Z. Metallkunde 40,54 [1949]. Bezügl. früherer Arbeiten über Rückbildung und Kaltaushärtung siehe das zusammenfassende Referat von H. B orchers, Metallwirtsch. Metallwiss., Metalltechn. 20, 1161 [1941]. sowie den Bericht in Fiat Review of German science, Non-ferrous Metallurgy I, S. 80 [1948]. 
dungszeit. Messungen der magnetischen Suszeptibilität führten, dank ihrer übersichtlichen Zuordnungsmöglichkeit zur Zustandsänderung, in systematischer Weiterentwicklung zu dem eigentlichen Rückbildungsverfahren, das durch quantitative Aufnahme und Auswertung der Rückbildungskinetik bis zur Berechnung von Aktivierungsenergien der einzelnen Zustände durchgeführt werden konnte.*

Das Rückbildungsverfahren kann vor allem auch dann angewandt werden, wenn eine Legierung aus mehreren Zuständen mit verschiedener thermischer Stabilität besteht. Die Existenzkurven dieser Zustände verlaufen ja gewöhnlich bei tieferen Temperaturen konstant (soweit die verminderte Diffusionsgeschwindigkeit überhaupt noch die völlige Ausbildung des Zustandes erwarten läßt) und fallen dann in einem für jeden Zustand charakteristischen Temperaturbereich auf Null ab. Sind die Temperaturen beginnender Instabilität, also der Abfall der Existenzkurven, genügend getrennt, so kann durch Temperatursteigerung jeder Zustand für sich rückgebildet und quantitativ erfaßt werden. Wenn jedoch die Abbaugebiete mehrerer Zustände sich teilweise überdecken, stellt die Analyse der Rückbildungskurven erhöhte Anforderungen an die Genauigkeit und Schnelligkeit nicht nur der Messung, sondern ebenso auch der Temperaturbehandlung der Proben. Im Verlauf von Untersuchungen mit schnellen Temperaturwechseln, die zunächst vor allem eine Erhöhung der Meßgenauigkeit und exaktere Versuchsbedingungen zum Ziele hatten, ergab sich ein nëuer Gesichtspunkt durch die Feststellung, daß der innere Mechanismus der Zustandsänderung wesentlich beeinflußt wird durch die Geschwindigkeit, mit der die Proben auf die Anlaßtemperatur gebracht werden.

Wie die folgenden Ergebnisse zeigen, können während der Temperung beim Aufheizen, je nach - der Geschwindigkeit des Durchlaufens bestimmter Temperaturgebiete, Atomanordnungen entstehen, die selbst zwar kaum eine unmittelbar

* Wir würden es bedauern, wenn die erfreuliche Klärung der Vorgänge, wie sie das Rückbildungsverfahren gebracht hat, durch die verschiedentlich angewandte Ausweitung des Begriffes ,Rückbildung“ auf die Wiederverfestigung, also die Bildung neuer Zustände, beeinträchtigt würde, und wir möchten, in Übereinstimmung mit W. Köster, entschieden empfehlen, die Bezeichnung „Rückbildung“ ausschließlich den rückläufigen Vorgängen zum ungeordneten Mischkristall hin vorzubehalten. meßbare Rückwirkung auf die untersuchte Eigenschaft haben, die aber von wesentlichem Einfluß auf die Vorgänge bei höheren Temperaturen, bei denen sie selbst längst nicht mehr existenzfähig sind, sein können.

Normales oder extrem schnelles Aufheizen (,Aufschrecken“) kann somit u. U. ebenso Verschiedenheiten für den weiteren Verlauf der $\mathrm{Zu}$ standsänderung zur Folge haben, wie der längst bekannte und beachtete Unterschied zwischen $\mathrm{Ab}$ kühlen und Abschrecken, wenn auch der innere Mechanismus dieses Einflusses natürlich ein anderer ist.

3. Das A ufschreckverfahren

Wir berichten im folgenden über magnetische und Härtemessungen an Proben, die durch eine besondere Anordnung sehr schnell und kurzzeitig in ihrer Temperatur geändert, also sowohl schnellem Aufheizen (Aufschrecken), wie auch kurzzeitigen Überhitzungen (Wärmestößen) unterworfen werden konnten. Die Entwicklung der Aufschreckapparatur mit Hochfrequenzheizung sowie die Durchführung eines Teiles der Härtemessungen ist der Mitarbeit von Hrn. G. W. Healy zu danken.

Bei den normalen Erwärmungsmethoden (Einbringen der Proben in einen vorgeheizten Gasraum oder ein ölbad) ist die Aufheizgeschwindigkeit durch den äußeren Wärmeübergang festgelegt und sinkt mit Annäherung an die gewünschte Anlaßtemperatur asymptotisch auf Null, so daß gerade die höheren Zwischentemperaturen, bei denen infolge der gesteigerten Atombeweglichkeit am meisten passieren kann, besonders langsam durchlaufen werden. Zum schnellen Aufheizen muß demnach eine starke Energiezufuhr, die bei dauernder Einwirkung die Probe in kurzem zum Schmelzen brächte, kurzzeitig einwirken, aber im Augenblick der erreichten Anlaßtemperatur unterbrochen werden. Gleichzeitig ist die Probe ohne Wärmeverlust in den vorgeheizten Anlaßofen zu verbringen.

Unter den verschiedenen Möglichkeiten einer schnellen Energiezufuhr haben sich zwei Anordnungen bewährt: 1. Die induktive Energiezufuhr durch - Hochfrequenzströme, 2. die unmittelbare Wärmezufuhr zur Probe bei kurzzeitigem Durchlaufen eines stark überheizten Röhrenofens vor dem Einbringen in den vorgeheizten Anlaßofen ${ }^{14}$.

14 Vgl. hierzu auch H. Borchers u. O. Castell a n i, Z. Metallkunde 35, 128 [1943]. 
Unter Verzicht auf die Wiedergabe technischer Einzelheiten der Apparaturen seien lediglich einige erwähnenswerte Besonderheiten angeführt: Die Proben sind einheitlich $12 \mathrm{~cm}$ lang bei $3 \mathrm{~mm} \varnothing$. Die ölgekühlte Hochfrequenzspule (200 bis $800 \mathrm{kHz}$ ), bzw. der Überhitzungsofen, der vorgeheizte Anlaßofen und das Abschreckgefäß hängen senkrecht untereinander, so daß durch schnelles, schrittweises Senken der aufgehängten Probe halbautomatisch Aufheiz- und Temperungszeiten von einigen Sekunden genau reproduzierbar eingestellt werden können. Zur Temperaturverfolgung ist je ein Thermoelementdraht in zwei getrennte achsenparallele Bohrungen der Probe eingeführt, so daß die Probenwand dazwischen einen Teil des Thermokreises bildet. Variable Ubergangswiderstände werden durch Kompensationsmessung eliminiert. Die Temperaturdifferenz zwischen Staboberfläche und Achse (auch bei Hochfrequenzheizung infolge Skin-Effekts) bleibt auf Grund von Messungen unter $2^{\circ}$.

\section{Vergleich verschiedener Aufheiz- verf a hren}

Nach Abb. 1 erreicht eine Probe beim Einbringen in einen auf $250^{\circ}$ vorgeheizten Röhrenofen nach der gewünschten Anlaßzeit von z. B. $60 \mathrm{sec}$ nur $230^{\circ}(1)$, im Ölbad erreicht sie $25 \mathrm{sec}$ nach dem Eintauchen die Endtemperatur (2), beim Auf-

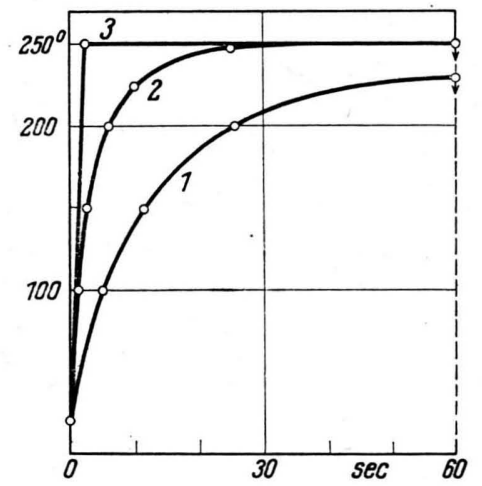

Ahb. 1. Aufheizgeschwindigkeit im Röhrenofen (1), im Ölbad (2) und beim Aufschrecken (3) mit Hochfrequenz.

schrecken (mit Hochfrequenz) schon nach 2,5 sec (3). Gleichzeitig ist den Aufheizkurven zu entnehmen, wie lange sich die Proben auf höheren Zwischentemperaturen befinden, bei denen $u$. U. ungewollte Zustandsänderungen vor sich.gehen können. So wird z. B. der Temperaturbereich von 100 bis $250^{\circ}$, in welchem im System Al-Cu erhebliche Zustandsänderungen ablaufen (Bildung und Rückbildung von Kalt- und Warmaushärtung), im Röhrenofen erst in mehr als 3 min, im
Ölbad in 24 sec durchlaufen, während beim ,Aufschrecken" die Probe sich weniger als 2 sec in diesem unerwünschten Temperaturintervall befindet. Die Meßbeispiele des Abschn. 6 zeigen. wie durch diesen Unterschied eine Zustandsänderung in sehr verschiedener Weise ablaufen kann.

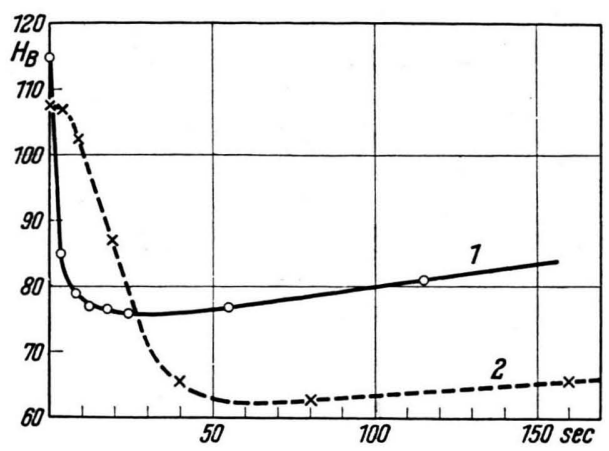

Abb. 2. Rückbildung der Kaltaushärtung einer abgeschreckten (1) bzw. abgekühlten (2) Probe (Mischkristall $\mathrm{Al}+5 \% \mathrm{Cu}$ ) durch Aufschrecken auf $200^{\circ}$.

\section{Kinetik schneller Zustands- änderungen}

Im übersättigten Mischkristall $\mathrm{Al}+5 \% \mathrm{Cu}(\mathrm{ab}-$ geschreckt von $550^{\circ}$ ) entsteht beim Anlassen auf Temperaturen zwischen Zimmertemperatur (ZT) und $150^{\circ}$ durch negative Diffusion eine Atomanordnung, die eine starke Vergütung der mechanischen Eigenschaften bewirkt, die bei Temperaturen über $200^{\circ}$ aber wieder vollständig und sehr schnell zerstört (rückgebildet) wird. Dieser Zusammenbruch der Kaltaushärtung bei $200^{\circ}$ innerhalb weniger Sekunden ist in Abb. 2, Kurve 1, an Hand von Härtemessungen in Anlaßstufen von je 4 sec wiedergegeben. Ausgangspunkt der Kurve ist die Kaltaushärtung nach 30 min Temperns auf $100^{\circ}$; der Wiederanstieg der Härte nach Durchlaufen des Minimums ist auf die Bildung eines bei $200^{\circ}$ stabilen Zustandes $b$ zurückzuführen. $Z$ wischen den einzelnen Meßpunkten bei ZT wird die Probe für die kurze Anlaßzeit auf $200^{\circ}$ aufgeschreckt.

Kurve 2 der Abb. 2 gibt die Rückbildung der Kaltaushärtung $\left(20 \mathrm{~h}\right.$ auf $\left.100^{\circ}\right)$ an einer von $550^{\circ}$ nicht abgeschreckten, sondern an Luft abgekühlten Probe. Infolge des Fehlens der Abschreckspannungen ist die Aushärtung trotz längeren Anlassens niedriger als bei der abgeschreckten Probe, aber auch die Rückbildung verläuft nunmehr mit Andeutung einer Inkubationszeit langsamer und zu tieferem Minimum. 
Die Warmaushärtung infolge des bei $215^{\circ}$ entstehenden Zustandes b kann bei Temperaturen über $270^{\circ}$ vollständig rückgebildet werden (Abb.3). Die Ausgangswerte der Kurven 1 und 2 sind nach 15 min bzw. $2 \mathrm{~h}$ Anlassens der abgeschreckten Proben auf $215^{\circ}$ gemessen. Entsprechend der

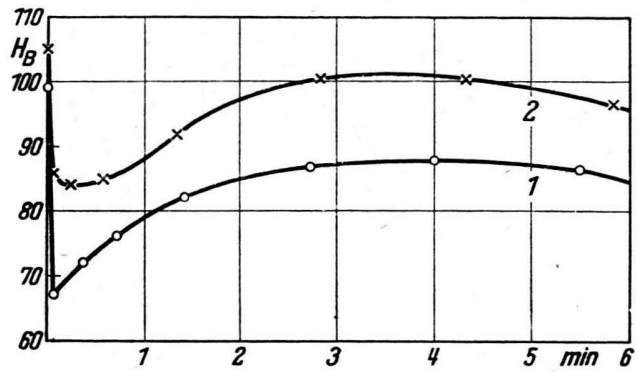

Abb. 3. Rückbildung der Warmaushärtung einer 15 Min. (1) bzw. 2 Stdn. (2) bei $215^{\circ}$ ausgehärteten Probe (Mischkristall $\mathrm{Al}+5 \% \mathrm{Cu}$ ) durch Aufschrecken auf $300^{\circ}$.

höheren Temperatur läuft die Rückbildung bei $300^{\circ}$ noch schneller als nach der Kaltaushärtung ab, die Temperungsintervalle von 2,5 sec reichen bei der, $2 \mathrm{~h}$ angelassenen Probe gerade noch zur Erfassung des Abfalles aus. Die verschiedene Höhe des Kurvenverlaufs weist darauf hin, daß während des längeren Anlassens ( $2 \mathrm{~h}$ auf $215^{\circ}$ ) vor der Rückbildung der Kurve 2 schon der Ausscheidungszustand c entsteht, der bei $300^{\circ}$ nicht mehr rückbildbar ist, sondern einen erneuten Härteanstieg veranlaßt, schließlich aber durch Koagulation ein Absinken der Härte verursacht.

Die genaue Aufnahme der Kinetik bis zu möglichst hohen Temperaturen ist für die Festlegung der logarithmischen Temperaturabhängigkeit, und damit für die Genauigkeit der Bestimmung von Aktivierungsenergien von Bedeutung.

Aus den Meßreihen der Abb. 2 und 3 können die thermischen Bedingungen zur Trennung der drei Zustände $a, b$ und $c$ entnommen werden, die aus dem übersättigten Mischkristall Al-Cu durch Kaltaushärtung, Warmaushärtung und Ausscheidung entstehen können.

6. Einfluß der Aufheizgeschwindigkeit auf den Verlauf der Zustandsänderung

Neben der Möglichkeit kurzzeitiger Temperung zur punktweisen Aufnahme schneller $\mathrm{Zu}^{-}$ standsänderungen gestattet die hohe Anheiz- geschwindigkeit der Aufschreckanordnung die Untersuchung und gegebenenfalls Ausschaltung von Atomzuständen, die während des langsameren Aufheizens bei den $Z$ wischentemperaturen entstehen und die weitere Zustandsänderung wesentlich beeinflussen können ${ }^{15}$. Der verschiedene Ablauf des Vorgangs, je nach der Aufheizgeschwindigkeit, geht aus den Abb. 4 und 5 hervor. In beiden Fällen sind die Proben vorher $16 \mathrm{~h}$ auf $550^{\circ}$ homogenisiert und abgeschreckt. Das Aufheizen auf die Anlaßtemperatur $215^{\circ}$ erfolgt aber in den Meßreihen der Abb. 4 verhältnismäßig langsam durch Einführen der Probe in einen Röhrenofen (vgl. Abb.1), während in den

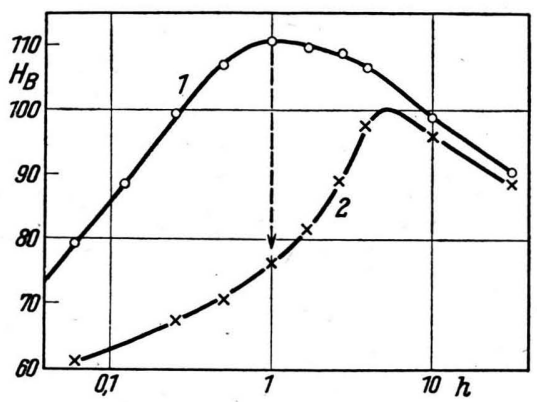

Abb. 4. Bildung (1) und Rückbildung (2) der Warmaushärtung einer nach langsamem Aufheizen auf $215^{\circ}$ ausgehärteten Legierung $(\mathrm{Al}+5 \% \mathrm{Cu})$.

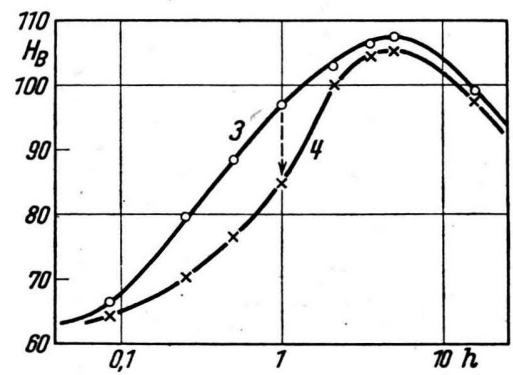

Abb. 5. Bildung (3) und Rückbildung (4) der Warmaushärtung einer auf $215^{\circ}$ aufgeschreckten Legierung $(\mathrm{Al}+5 \% \mathrm{Cu})$.

Kurven der Abb. 5 der Bereich von Zimmertemperatur bis $215^{\circ}$ in 2,5 sec durchlaufen wird. Obwohl die Anheizzeit bei der Anlaßdauer miteingerechnet ist, die langsam aufgeheizte Probe sich

15 Vgl. hierzu auch die Mitt. von W. Bungardt u. V. Hauk, Metallforschg. 2 bzw. Z. Metallkunde 38, 161 [1947], wonach gegensätzliche Versuchsergebnisse in der Aushärtharkeit von Al-Zn-Mg-Legierungen (W. Feldmann, Metallwirtsch. Metallwiss., Metalltechn. 20, 501 [1941]) durch den Einfluß der Erhitzungsgeschwindigkeit gedeutet werden können. 
also im ganzen kürzere Zeit auf der Anlaßtemperatur befand, ist hier die Warmaushärtung gegenüber der aufgeschreckten Probe fast um das Fünffache beschleunigt, so daß z. B. das Maximum nach $1 \mathrm{~h}$ gegenüber $5 \mathrm{~h}$ erreicht wird. Daß die bei

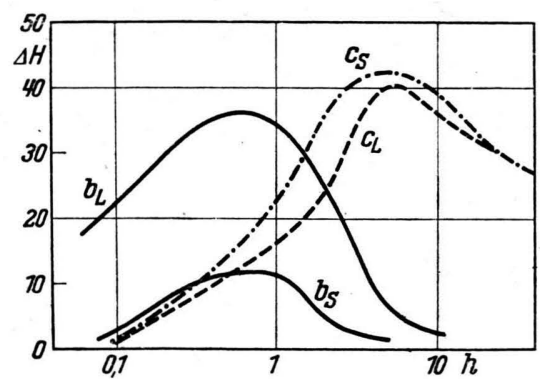

Abb. 6. Rückbildungsanalyse der Zustände b und c nach langsamem Aufheizen $\left({ }^{b}{ }_{L}, c_{L}\right)$ und nach Aufschrecken $\left(b_{S}, c_{S}\right)$.

verschiedener Anheizgeschwindigkeit entstehenden Zustände aber auch bezüglich ihrer thermischen Stabilität verschieden aufgebaut sind, wird deutlich, wenn man von den einzelnen Meßpunkten der Kurven 1 und 3 aus Rückbildungen, z. B. durch Aufschrecken auf $300^{\circ}$, durchführt. Der übrigbleibende Betrag der Härtesteigerung (2 bzw. 4) gibt ein $\mathrm{Ma}$ für den jeweiligen Anteil des bei $300^{\circ}$ noch stabilen Zustandes c (heterogene Ausscheidung, nicht weiter rückbildbar).

In der Abb. 6 ist die Differenz der Kurven 1-2 bzw. 3-4 als Maß für die Menge des bei $215^{\circ}$ entstandenen und bei $300^{\circ}$ rückbildbaren Zustandes $b$ den Ausscheidungskurven $c$ gegenübergestellt. Es wird deutlich, wie die Beschleunigung der Warmaushärtung bei langsamem Aufheizen ausschließlich auf die vermehrte b-Bildung (Kurve $b_{L}$ ) zurückzuführen ist, während die Ausscheidung von c (Kurve $c_{L}$ ) sogar etwas verzögert wird. Der wesentlich größere Anteil von b nach langsamem Aufheizen kann jedoch nicht damit erklärt werden, daß sich dieser Zustand während des Durchlaufens der Zwischentemperaturen bildet; seine Entstehungsgeschwindigkeit ist ja in diesem Bereich viel kleiner, als wenn die Probe sofort auf $215^{\circ}$ gebracht wird. Aber es liegt nahe, zu vermuten, daß beim langsamen Durchlaufen der Zwischentemperaturen Übergangszustände der Atomanordnung $b$ entstehen, die selbst noch keine starke Rückwirkung auf die untersuchte Eigenschaft haben (Kohärenzbereich!), aber als vorbereitende oder auslösende Keime die folgende
b-Bildung unterstützen, ja u. U. erst ermöglichen. Vielleicht würde bei noch schrofferem Aufschrekken der b-Zustand überhaupt nicht auftreten und die c-Ausscheidung noch früher einsetzen. Die mit starker b-Bildung gekoppelte Verzögerung der Ausscheidung bei langsamem Aufheizen zeigt, daß der b-Zustand nicht als vorbereitender Zwischenzustand die Ausscheidung beschleunigt. Unsere schon vor langem ausgesprochene Vermutung ${ }^{5}$. daß es sich bei der Kalt- und Warmaushärtung um Nebenzustände, gewissermaßen Sackgassen der Atomanordnung handelt, aus denen der Weg zur heterogenen Ausscheidung u. U. sogar wieder zurück über den ungeordneten Mischkristall führt. scheint in diesen Ergebnissen eine neue Bestätigung zu finden.

Die beiden Nebenzustände unterscheiden sich jedoch sehr bezuiglich ihrer thermischen Stabilität. Der Zustand a der Kaltaushärtung hat eine einheitliche Bindungsfestigkeit mit konstanten Aktivierungsenergien für Bildung und Rückbildung, unabhängig von der Temperatur, bei der er entstanden ist. Seine Existenzkurve kann reversibel durchlaufen werden. Seine Eigenschaftsänderung z. B. bei der Suszeptibilität, läuft in Richtung einer örtlichen Anreicherung der Fremdatome im Mischkristall (negative Diffusion) in

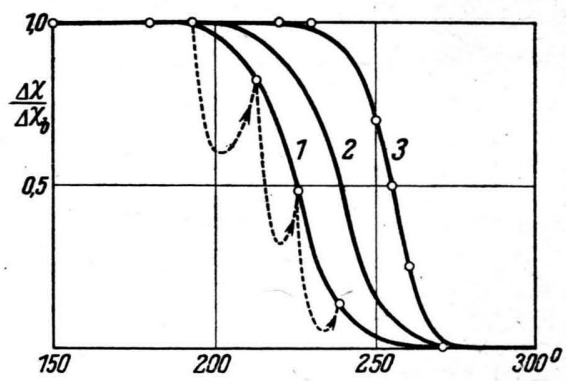

Abb. 7. Relative Suszeptibilitätsänderung bei Rückbildung einer langsam aufgeheizten (1), einer aufgeschreckten (2) und mit darauffolgendem Wärmestoß (3) ausgehärteten Legierung $(\mathrm{Al}+5 \% \mathrm{Cu})$.

Übereinstimmung mit neueren röntgenographisehen Befunden, die eine plattenförmige Anordnung der Fremdatome im Grundgitter wahrscheinlich machen ${ }^{16}$.

10 G. D. Preston, Proc. physik. Soc. 52, 77 [1940]: J. Cálvet, P. Jaquet u. A. Guin ier, C. R. hebd. Séances Acad. Sci. 206, 1972 [1938], J. Instn. Met. 65, 121 [1940], ref. in Metallwirtsch. Metallwiss. Metalltechn. 21, 433 [1942]; ferner G. W a s s e r m a n n, Metallwirtsch. Metallwiss., Metalltechn. 23, 387 [1944]. 
Demgegenüber ist der Nebenzustand b weitgehend uneinheitlich. Die Logarithmen der Bildungs- und Rückbildungsgeschwindigkeiten liegen im Diagramm gegen $1 / T$ nicht auf Geraden; die Berechnung einer einheitlichen Aktivierungsenergie wird damit illusorisch. Statt der Reversibilität tritt bei schnellen Temperaturänderungen im Wechselspiel von Bildung und Rückbildung eine ausgesprochene Hysterese zwischen den verschieden stabilen Anteilen auf. Für die Deutung dieser komplizierteren Zustandsänderungen sind die Ergebnisse von Suszeptibilitätsmessungen auf Grund ihrer einfachen, monotonen $\mathrm{Zu}$ ordnungsmöglichkeit zur Zustandsmenge besonders geeignet ${ }^{17}$. An Hand von Abb. 7, in der als Ordinate die relative Suszeptibilitätsänderung, bezogen auf die jeweils durch b bewirkte Gesamtänderung $\Delta \chi_{\mathrm{b}}$ aufgetragen ist, lassen sich die charakteristischen Merkmale des Zustandes b in drei Punkten zusammenfaśsen.

1. Die Existenzkurve kann nur in Richtung steigender Temperatur abgebaut werden. Beim schnellen Ubbergang auf eine höhere Temperatur sinkt die Suszeptibilität zunächst sehr schnell auf ein Minimum, um dann etwas langsamer wieder zur Abbaukurve anzusteigen ${ }^{18}$. Nach erfolgter Rückbildung kann die Existenzkurve nicht rückwärts durchlaufen werden, vielmehr setzt erst nach erheblicher „Unterkühlung“ eine Neubildung von b in geringem Maße ein.

2. Der Verlauf der Abbaukurve ist abhängig von den Entstehungsbedingungen der Zustände. Die Kurve verschiebt sich um so weiter nach rechts (2), d. h. die Zustände sind um so stabiler, je höher ihre Bildungstemperatur und je schneller die Aufheizgeschwindigkeit war (letzteres unter Verminderung der Gesamtmenge von b).

3. Die stärkste Verschiebung der Existenzkurven zu höheren Temperaturen (3) wird durch sehr kurzzeitiges Überhitzen über den Stabilitätsbereich der Zustände hinaus (Wärmestoß über $270^{\circ}$ ) bewirkt.

17 Obwohl die Härte unter den mechanischen Eigenschaften die übersichtlichste Zuordnung zu den $\mathrm{Zu}-$ standsmengen aufweist, zeigt doch ihre Wiederabnahme nach längerem Tempern, die ja natürlich nicht auf eine Ausscheidungsverminderung, sondern auf Koagulation zurückzuführen ist, daß in diesen Gebieten eine Mengenanalyse nur durch Vergleich mit einer vom Gefüge unbeeinflußten Eigenschaft, z. B. der Suszeptibilität, möglich ist.
Diese Ergebnisse sind zwanglos vereinbar mit der Auffassung, daß der Zustand b sich aus einer mehr oder weniger kontinuierlichen Reihe von Atomgruppierungen mit steigender thermischer Stabilität zusammensetzt. In Übereinstimmung mit der von R. Becker entwickelten Keimbildungstheorie in Mischkristallen bilden sich bei niedrigeren Temperaturen bevorzugt kleinere oder schwächer gebundene Komplexe, die aber bei Temperatursteigerung schnell wieder zerschlagen (rückgebildet) werden, sobald sie kleiner als die mit der Temperatur stark anwachsende ,kritische Keimgröße" sind. Da diese Rückbildung schneller verläuft als sich die der neuen Temperatur entsprechenden stabileren Komplexe mit höherer Aktivierungsenergie bilden können, ergibt sich der in den gestrichelten Pfeilen der Abb. 7 angedeutete Verlauf.

Abschließend noch eine kurze Bemerkung im Hinblick auf neuere röntgenographische Untersuchungen der Aushärtung. Wir haben im Vorstehenden die für Rückbildungsergebnisse eingeführte Bezeichnung der Aushärtungszustände $a, b$ und $c$ angewandt, während in röntgenographischen Arbeiten gewöhnlich von der Aushärtungsphase $\Theta^{\prime}$ und der Ausscheidung $\Theta$ gesprochen wird. In diesem formalen Unterschied kommt letztlich eine noch ungelöste, grundsätzliche Verschiedenheit der Auffassungen zum Ausdruck. Nach den Röntgenergebnissen, die über die geometrische Anordnung der Atome, nicht aber bisher über Menge und Stabilität der Phasen quantitativen Aufschluß geben, erscheinen die einzelnen Aushärtungszustände als kontinuierlicher Utbergang vom Mischkristall über die einphasige Entmischung zur Aushärtungsphase $\Theta^{\prime}$ und schließlich zur Ausscheidung $\Theta-$ jeder Zustand als vorbereitende Vorstufe des folgenden ${ }^{19}$. Demgegenüber sprechen die Rückbildungsmessungen an mengenmäßig auswertbaren Eigenschaftsänderungen immer wieder für eine mehr.oder weniger selbständige Existenz der einzelnen Aushärtungszustände, die nebeneinander herlaufen können, ohne durch ihr Vorhandensein das Auftreten eines anderen Zustandes wesentlich zu erleichtern oder

18 Die gestrichelten Pfeile in Abb. 7 sollen den zeitlichen Verlauf der Suszeptibilität beim schnellen Ǔbergang auf eine höhere Temperatur andeuten, sie sind nicht den Abszissen der $\mathrm{Zw}$ ischentemperaturen zuzuordnen.

19 Vgl. z. B. G. W as s e r mann, Metallwirtsch. Metallwiss. Metalltechn. 23, 391 [1944]. 
örtlich vorzubereiten ${ }^{20}$. Eine Koordinierung dieser verschiedenen Auffassungen könnte wohl, wenigstens von der experimentellen Seite, am ehesten erreicht werden, wenn parallel laufende Untersuchungen mit den verschiedenen Methoden, aber mit gleichen Wärme- und Rückbildungs- behandlungen durchgeführt und die verschiedenen Gesichtspunkte vergleichbar gegeneinander abgewogen werden könnten.

20 S. Anm. 5-7, ferner H. Borcher ${ }^{13}$ sowie $u$. a. A. Durer u. W. Köster, Z. Metallkunde 30, 311 [1938].

\title{
Die Atomanordnung des amorphen Germanium
}

\author{
Von Otto Fürst, Richard Glocker und Hans Richter \\ Aus dem Röntgeninstitut der Technischen Hochschule Stuttgart \\ und dem Institut für Metallphysik am Max-Planck-Institut für Metallforschung, Stuttgart \\ (Z. Naturforschg. 4 a, 540-542 [1949]; eingegangen am 28. März 1949)

\begin{abstract}
Die Atomverteilung in amorphem, durch Aufdampfen hergestelltem Germanium wird die Zahl der Nachbaratome und ihre radialen Abstände vom betrachteten Atom mit denen des Gitters überein. Es wird ein Modell angegeben, das den Zusammenhang
\end{abstract} \\ durch eine Röntgen-Fourier-Analyse bestimmt und als verschieden von der des flüssigen \\ Germanium (Hendus) gefunden. Für die beiden ersten Koordinationsgruppen stimmen \\ zwischen der Atomanordnung des Gitters und der des amorphen Zustandes erklärt.
}

$\mathrm{D}^{\mathrm{i}}$ e Kenntnisse von der Atomanordnung in flüssigen und amorphen Stoffen ${ }^{1}$ haben durch die Röntgen-Fourier-Analyse ${ }^{2}$ eine starke Erweiterung erfahren. Beobachtungen an der amorphen und an der flüssigen Phase desselben Stoffes liegen bisher nicht ${ }^{3}$ vor; sie könnten Anhaltspunkte für die Ermittlung der gesetzmäßigen Verknüpfung der Atomverteilungen in den verschiedenen Phasen liefern. Von den verschiede-

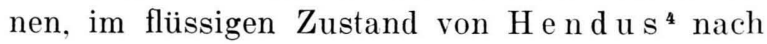
der Fourier-Methode untersuchten Elementen erscheint Germanium für einen solchen Vergleich besonders geeignet. Die Atomanordnung des flüssigen Germanium ist verschieden von der des kristallinen; jedes Atom ist in erster Koordination von 8 Nachbarn umgeben, statt von 4 im Gitter. Wie gleichzeitig aufgenommene Elektroneninterferenzbilder von $\mathrm{H}$. Richte ${ }^{5}$ zeigen, existiert auch eine amorphe Phase, deren Streubild von dem der flüssigen Phase sich deutlich unterscheidet. Die durch Aufdampfen im Hochvakuum gewonnenen Schichten haben günstigstenfalls Dicken von $1 \cdot 10^{-4} \mathrm{~cm}$, die zu Röntgenaufnahmen bei weitem nicht ausreichen.

Nach verschiedenen Versuchen ist es neuer-

${ }^{1} \mathrm{Vgl}$. den zusammenfassenden Bericht von R. G lokke r, Ergebn. exakt. Wiss. 22, 186 [1949].

2 F. Zernike u. J. Prins, Z. Physik 41, 184 [1927] ; P. Debye u. H. Menke, Physik. Z. 31, 797 [1930]; B. E. Warren u. N. S. Gingrich, Physic. Rev. 46, 368 [1934]. dings gelungen, dünne, auf Steinsalz aufgedampfte Germaniumhäutchen abzulösen und so auf einem geeigneten Träger aufeinanderzupacken, daß sich eine zur Entstehung von Röntgeninterferenzen ausreichende Schichtdicke ergab. An solchen Präparaten wurde mit kristallmonochromatisierter Strahlung der Wellenlänge 1,54 ̊ die Winkelabhängigkeit der Streuintensität photographisch aufgenommen und in bekannter Weise mittels Fourier-Analyse ausgewertet.

Die Winkelabhängigkeit der in bezug auf Absorption, Polarisation und inkohärente Strahlung korrigierten Streuintensität zeigt beim amorphen Germanium (Abb. 1) einen ganz anderen Verlauf als beim flüssigen (Abb. 3).

Demgemäß liefert auch die Fourier-Analyse in beiden Fällen wesentlich verschiedene Atomanordnungen. Die Größe $W$ in den Abb. 2 und Abb. 4 ist die Wahrscheinlichkeit, ein Atom im Abstand $r$ bis $r+d r$ vom betrachteten Ausgangsatom anzutreffen. Die regellose Atomverteilung,

3 Bei Selen scheinen die Röntgenstreubilder für den flüssigen Zustand ( $\mathrm{Pr}$ ins) und für den amorphen (K. Lark-Horovitz u. Miller) übereinzustimmen. Eine Bestimmung der Atomverteilung liegt nur im letzteren Falle vor (K. Lark-Horovitz u. E. P. Miller, Physic. Rev. 51, 380 [1937] bzw. H. Hend us, Z. Physik 119, 265 [1942].

4 H. Hendus, Z. Naturforschg. 2a, 505 [1947].

5 H. Richter, nach unveröffentlichten Versuchen aus dem Jahre 1943; ferner H. König, Optik 3, 201 [1948]. 